\title{
Acute lupus pneumonitis followed by intestinal pseudo-obstruction in systemic lupus erythematosus: A case report
}

\author{
CAIHONG $\mathrm{JI}^{1}$, XING YU ${ }^{2}, \mathrm{YONG}^{\mathrm{WANG}}{ }^{2}$ and LUFENG SHI $^{1}$ \\ Departments of ${ }^{1}$ Neurology and ${ }^{2}$ Surgery, The Second Affiliated Hospital, \\ Zhejiang University School of Medicine, Hangzhou, Zhejiang 310009, P.R. China
}

Received February 4, 2015; Accepted March 18, 2016

DOI: $10.3892 /$ etm.2016.3316

\begin{abstract}
Intestinal pseudo-obstruction (IpsO) and acute lupus pneumonitis (ALP) are uncommon severe complications of systemic lupus erythematosus (SLE). The present study reports the case of a 26-year-old female who presented with abdominal pain, nausea and vomiting as initial symptoms. Computed tomography (CT) scanning revealed the jejunal wall was thickened and streaky, mimicking the presentation of intestinal obstruction. Following emergency surgery, the patient's general condition was aggravated, with evident limb erythematous rashes. A series of laboratory examinations revealed SLE, and combined with patient's medical history IpsO was diagnosed, with a disease Activity Index score of 10. During the therapeutic period, high fever, dyspnea and oxygen saturation $\left(\mathrm{SaO}_{2}\right)$ reductions were detected, and CT scans indicated lung infiltration, excluding other causes through a comprehensive infectious work-up and a bronchoalveolar lavage examination. ALP was confirmed and treated with high-dose methylprednisolone and gamma globulin supplement. The patient responded well and was discharged in 2 weeks. In the one-year tapering period and after stopping corticosteroids, the patient recovered well with no relapse detected. In conclusion, the manifestation of IpsO in SLE is rare and represents a challenge for the surgeon to establish the correct diagnosis and avoid inappropriate surgical intervention. ALP may be the consequence of emergency surgery, and immediate high-dose glucocorticoid therapy is recommended.
\end{abstract}

Correspondence to: Dr Xing Yu or Dr Yong Wang, Department of Surgery, The Second Affiliated Hospital, Zhejiang University School of Medicine, 88 Jiefang Road, Hangzhou, Zhejiang 310009, P.R. China

E-mail: yuxing@zju.edu.com

E-mail: yx6808@163.com

Key words: systemic lupus erythematosus, intestinal pseudo-obstruction, emergency surgery, acute lupus pneumonitis, glucocorticoid

\section{Introduction}

Intestinal pseudo-obstruction (IpsO) is defined as ineffective intestinal propulsion which occurs in the absence of mechanical or obstructive factors (1). Cases, in whom IpsO is the primary clinical feature, are typically associated with connective tissue diseases. However, IpsO presenting as the first manifestation in systemic lupus erythematosus (SLE) is rare, and $<30$ cases of IpsO in patients with SLE have been reported (1-4). Clinical features generally include severe abdominal symptoms, such as distension, pain, nausea and vomiting, while radiographic signs show gaseous small bowel distension with air-fluid levels. Acute lupus pneumonitis (ALP) is an uncommon pulmonary manifestation of SLE, and lung involvement has only been reported in 3\% of SLE patients $(5,6)$. ALP is an unusual but life threatening complication of SLE, and usually presents with the acute onset of fever, cough, tachypnea and hypoxia. The radiological sign of ALP is consolidations in one or more areas, and is often associated with pleural effusion and pulmonary arterial hypertension. The majority of ALP responds to steroids and some requires treatment with cyclophosphamide; however, the rate of mortality remains high (5,7). IpsO and ALP occurring in a single patient, described as co-manifestation with SLE, has not been reported previously. Herein, we report a case in which IpsO was the initial presentation of SLE, and secondary onset of ALP occurred after emergency surgery.

\section{Case report}

A 26-year-old female, complaining of abdominal pain, nausea, vomiting and constipation for 1 day, was admitted to the emergency room of the Second Affiliated Hospital of Zhejiang University (Hangzhou, China) in June 2013. Written informed consent was obtained from the patient. Physical examinations showed distress countenance, soft abdomen, absence of bowel sounds, abdominal tenderness but no rebound tenderness. The auxiliary examinations of chest X-ray (Philips Medical Systems B.V., Eindhoven, The Netherlands) (Fig. 1A), white blood cell (WBC) count, hemoglobin (HGB), erythrocyte sedimentation rate (ESR), C-reactive protein (CRP) and urinalysis were normal. Computed tomography (CT) scanning showed that the jejunal wall was thickened and streaky (Fig. 1B and C), and a small quantity of ascites was observed. 

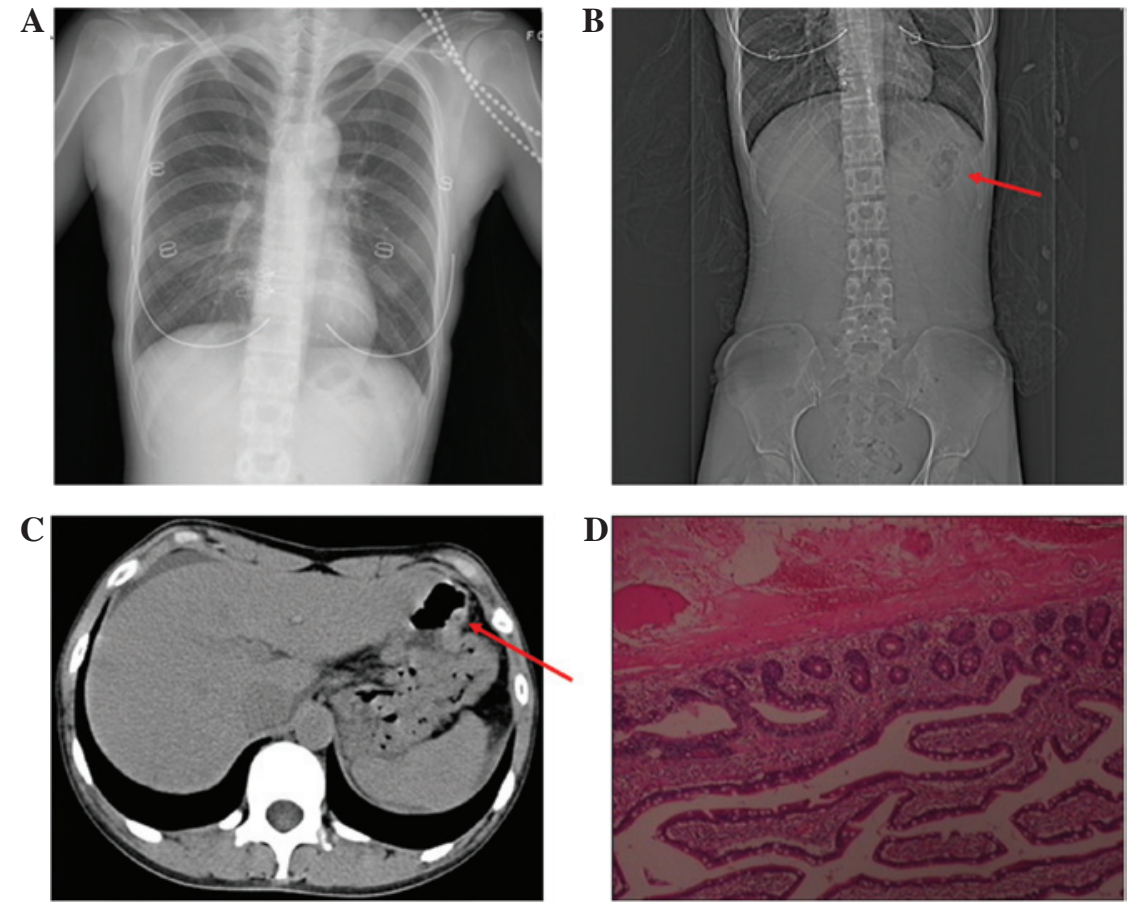

Figure 1. Radiological and pathological images of the patient during the first hospitalization. (A) Chest X-ray was normal. (B and C) Computed tomography scan showed sporadic jejunal wall thickening with streakiness (arrows). (D) Thickened jejunal wall (stain, hematoxylin \& eosin; magnification, x100).

The patient denied any history of hereditary, infectious or autoimmune diseases. The patient was thought to have strangulated intestinal obstruction, and an emergency laparotomy was performed. During the operation, $\sim 80 \mathrm{~cm}$ of the jejunum showed hyperemia and edema, with multiple petechiae on the serosal surface, and lymph node enlargement in the mesentery. In addition, $\sim 800 \mathrm{ml}$ turbid peritoneal fluid was detected, but no bowel perforation. Peritoneal lavage, segmental resection of the diseased jejunum and placement of a jejunostomy fistula were performed. Culture of the peritoneal fluid for bacteria and atypical bacteria was negative. Pathological examination of the jejunum using hematoxylin (Shanghai Bioscience \& Technology Co., Ltd., Shanghai, China) and eosin (Shanghai SSS Reagent Co., Ltd., Shanghai, China) (H\&E) staining showed chronic inflammation of intestinal mucosa, with submucosal anapetia, congestion, hemorrhage and interstitial edema. The muscle layer showed atrophy, hemorrhage, serosal congestion and edema (Fig. 1D).

Post-operatively, intestinal peristalsis did not recover, and a gastrointestinal decompression tube was placed in the duodenum. Apart from the bowel problem, sporadic rashes and mild fever without clear origin appeared. Following anti-infective treatment (ceftazidimine, $2.5 \mathrm{~g}$ intravenous injection, q.8.h; Hainan Haling Chemipharma Corporation, Ltd., Haikou, China) and careful nursing with electrocardiograph monitoring (MP 30; Philips Medical Systems B.V.) for 10 days, the patient demonstrated clinical improvement (abdominal pain was relieved, and the patient experienced no chills, nausea or vomiting, and vital signs were stable) and was discharged.

After 5 days, the patient was readmitted due to high fever $\left(39.2^{\circ} \mathrm{C}\right)$, upper abdominal pain, nausea and vomiting, particularly after a fatty meal. Abdominal X-ray and CT scanning (The Somatom Sensation 16; Siemens AG, Munich, Germany) revealed sporadic air-fluid levels and intestinal wall thickness (Fig. 2). Anti-infective treatment (cefoperazone and sulbactam, both $1.5 \mathrm{~g}$ intravenous infection, q.12.h; Pfizer, Inc., New York, NY, USA) was administered, but was ineffective. Multiple rashes on the lower extremities appeared on the fourth day, and laboratory examinations showed a rheumatoid factor of $20.4 \mathrm{IU} / \mathrm{ml}$ (increased), antinuclear antibody 1:320 (increased); ESR $26.00 \mathrm{~mm} / \mathrm{h}$ (increased); anti-ribonucleoprotein antibody +++ (strong), anti-Smith antibody +++ (strong), anti Sjogren's syndrome A antibody +++ (strong) and anti-Sjogren's syndrome B antibody + (weak). Antibodies against double stranded-DNA was not detected; complement (C3), $0.39 \mathrm{~g} / 1$ (decreased); CRP, $7.0 \mathrm{mg} / 1$ (normal); WBC, $2.5 \times 10^{9} / 1$ (decreased) and HGB, $89 \mathrm{~g} / 1$ (decreased). Furthermore, the patient had experienced hair loss and multiple red rashes in response to strong sunlight the previous year. SLE and lupus-related IpsO were then diagnosed according to American College of Rheumatology criteria (1997) (8), and the Systemic Lupus Erythematosus Disease Activity Index (SLEDAI) score was 10 (8). The patient was treated with $2 \mathrm{mg} / \mathrm{kg} /$ day methylprednisolone (Pfizer, Inc.) and her general condition improved after 2 days.

However, the patient had sudden onset of fever $\left(40.4^{\circ} \mathrm{C}\right)$ and dyspnea, oxygen saturation $\left(\mathrm{SaO}_{2}\right)$ was $87 \%$, and arterial blood gas analysis revealed a $\mathrm{pH}$ of $7.409, \mathrm{PCO}_{2}, 33.6 \mathrm{mmHg}$ and $\mathrm{PO}_{2}, 83.9 \mathrm{mmHg}$. Further laboratory examination showed: Glutamic pyruvic transaminase, $436 \mathrm{U} / 1$ (increased); glutamic oxaloacetic transaminase, $740 \mathrm{U} / \mathrm{l}$ (increased); $\mathrm{K}^{+}, 3.36 \mathrm{mmol} / 1$ (decreased); and $\mathrm{WBC}, \mathrm{HGB}, \mathrm{CRP}$ and urinalysis were normal. On chest $\mathrm{CT}$, both lungs showed ground-glass appearance, with uneven density, interlobular septal thickening and bilateral hydrothorax (Fig. 3A and B). Anti-infective treatment (imipenem and cilastatin, $0.5 \mathrm{~g}$ intravenous injection, q.8.h) was administered for 3 days; however, fever and dyspnea were 

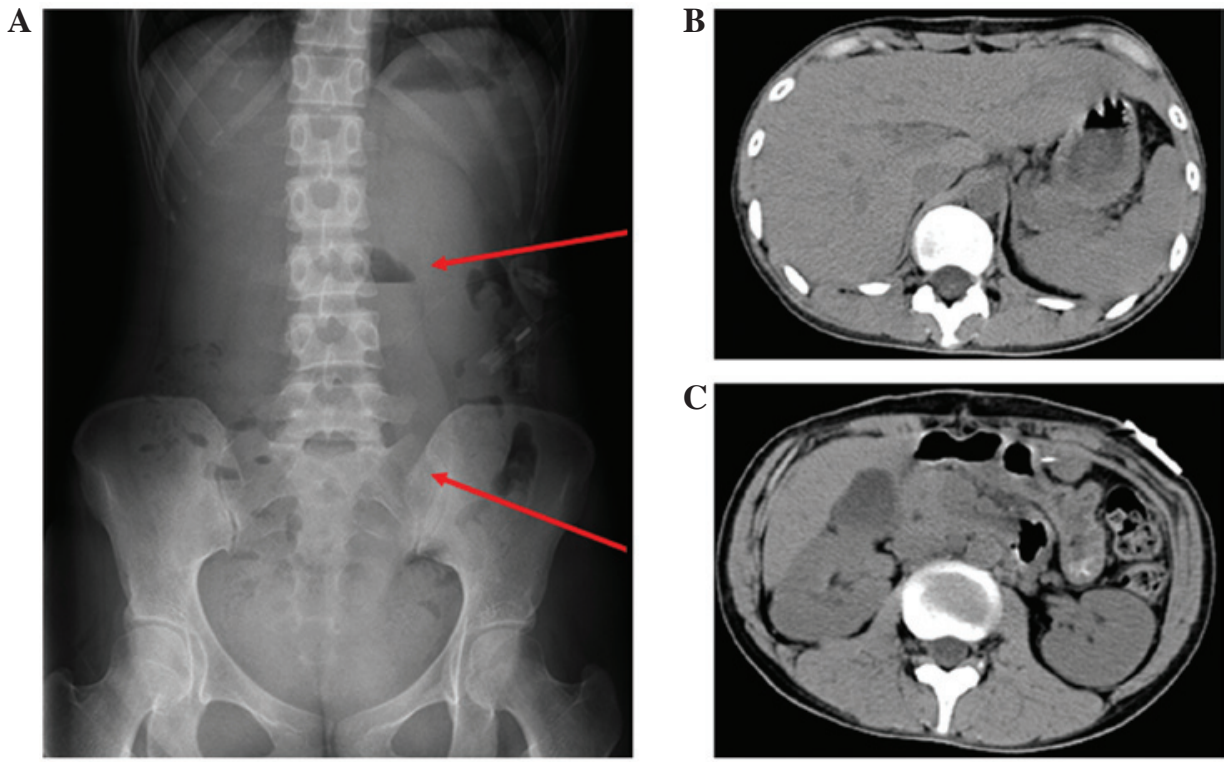

Figure 2. Radiological and pathological findings of the patient when the symptoms of intestinal pseudo-obstruction recurred. (A) Supine abdominal erect X-ray showed sporadic air-fluid levels (arrows). (B and C) Computed tomography scan showed sporadic air-fluid levels and intestinal wall thickness.

$\mathbf{A}$
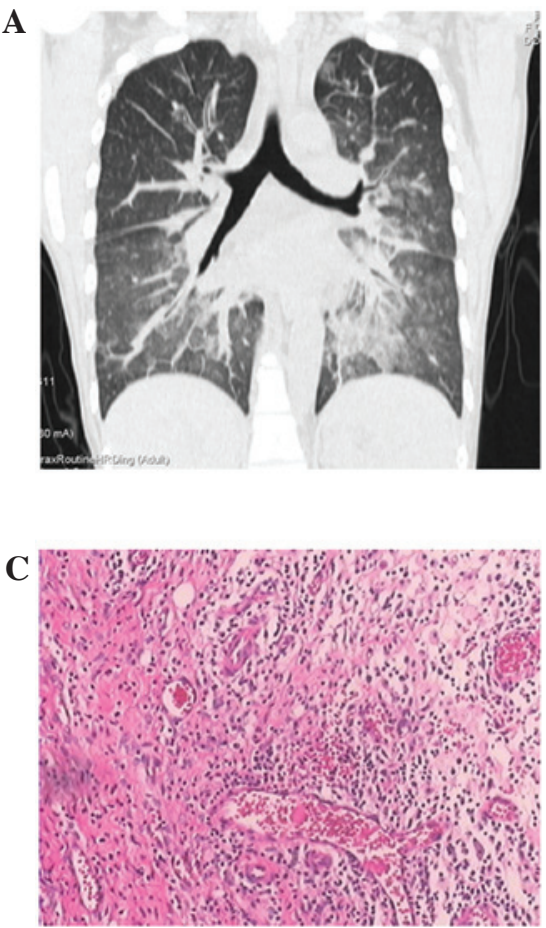

B

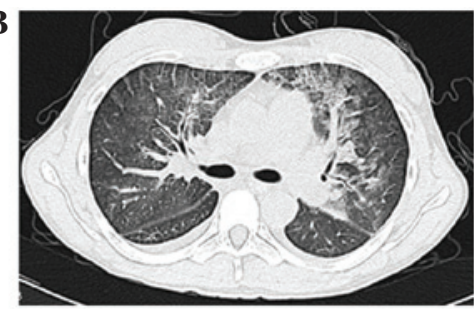

D

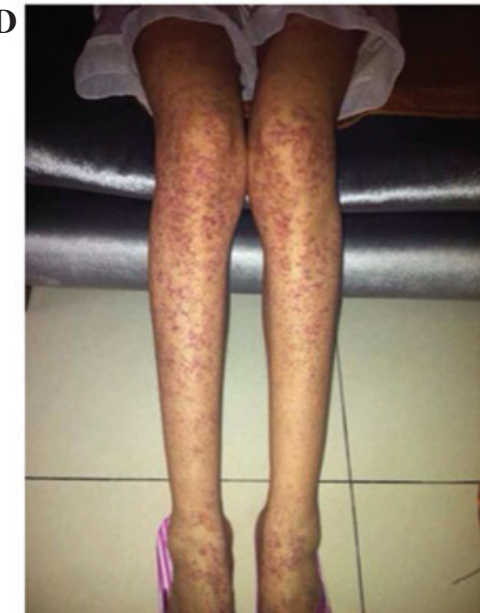

Figure 3. Radiological and pathological findings of the patient with acute lupus pneumonitis. (A and B) Computed tomography scan showed ground-glass appearance, with uneven density, interlobular septal thickening and bilateral hydrothorax in the lungs. (C) Pathological examination showed subcutaneous fibrous tissue hyperplasia with inflammatory cell infiltration (stain, hematoxylin \& eosin; magnification, x100). (D) Multiple erythematous rashes on the lower extremities.

not alleviated. A comprehensive infectious work-up (blood, sputum and urine cultures) was negative. A bronchoalveolar lavage (BAL; Olympus BX43; Olympus Corporation, Tokyo, Japan) examination from the affected areas was also negative for gram positive/negative bacteria, viral infections, fungal infections, acid fast bacilli or hemosiderin-laden macrophage. The erythematous rash was accentuated, and pathological biopsy results (using H\&E staining) showed subcutaneous fibrous tissue hyperplasia with inflammatory cell infiltration
(Fig. 3C and D). ALP was finally diagnosed and high-dose (1 g/day) methylprednisolone + gamma globulin therapy was administered. After 3 days treatment, the patient's temperature dropped to $37.0^{\circ} \mathrm{C}$ and dyspnea was significantly ameliorated. This therapy was followed by $2 \mathrm{mg} / \mathrm{kg} / \mathrm{day}$ methylprednisolone as maintenance treatment, and glycyrrhizin (Minophagen Pharmaceutical Co., Ltd., Tokyo, Japan) to improve liver function. Repeat chest CT scan showed significant improvement. 
During long-term tapering period or after stopping corticosteroids (methylprednisolone, Pfizer, Inc.), the patient recovered well with no relapse detected.

\section{Discussion}

The present study presents a rare case in which $\mathrm{IpsO}$ was the initial presentation of SLE with secondary onset of ALP after emergency surgery. To the best of our knowledge, there have been no previous reports of IpsO and ALP occurring in a single patient.

IpsO is defined as the presence of clinical features suggesting intestinal obstruction, but without organic obstruction (9). As in the present case, the clinical manifestations of IpsO include abdominal pain, nausea, vomiting, absence of bowel sounds and may include constipation and fever (4). An $\mathrm{X}$-ray and CT scan frequently show air-fluid levels and thickening of the small and/or large intestinal wall. IpsO may be the first manifestation of SLE in some patients; however, it usually represents a complication, with few case series described in the literature $(1,2,10)$. IpsO is easily confused with mechanical intestinal obstruction and represents a diagnostic challenge for the surgeon. Ureterohydronephrosis is highly associated with IpsO in SLE $(1,4,10)$, as repeated urinalysis examination results were negative, ureterohydronephrosis did not occur in this patient.

The pathophysiology of IpsO secondary to SLE remains unclear (9), although the hypotheses have suggested dysmotility due to a primary myopathy or secondary to an immune complex mediated vasculitis (11). The pathological characteristics of the gastrointestinal tract in chronic intestinal pseudo-obstruction (CIPO) patients include widespread myocyte necrosis, severe atrophy and fibrosis in the muscularis layer; active serositis with serosal thickening and fibrosis, with little or no evidence of vasculitis or injury $(11,12)$. In the present AIPO case, the pathological results of the jejunum showed muscle layer atrophy, with submucosal anapetia, congestion and hemorrhage. No evidence of vasculitis or thromboembolism was detected. Therefore, the present case supports the hypothesis that the clinical features of AIPO in SLE patients appear to be the result of a primary intestinal smooth muscle myopathy, similar to that in CIPO.

ALP is an uncommon (1-12\%) manifestation, but may be a life-threatening complication of SLE (12). The clinical features of ALP are characterized by acute onset of fever, cough, pleurisy and dyspnea. The key radiographic manifestation in the majority of cases is pulmonary infiltration, which appears on CT scan as ground-glass or honeycomb appearance $(5,13)$. The diagnosis of ALP is essential to exclude other causes of lung infiltration, particularly infective pneumonia (7). In the present case infective pneumonia was excluded, as a comprehensive infectious work-up was negative and anti-infective treatment was ineffective. Diffuse alveolar hemorrhage was excluded by the negative results of hemosiderin-laden macrophage in BAL fluid. ALP was finally diagnosed by the syndrome of acute reversible hypoxemia, the abnormal results of CT scan, and the exclusion of other causes of lung infiltration. The pathophysiology of ALP is based on an acute injury to the alveolar capillary units in pulmonary vasculature (7). ALP typically occurs during the active stage of SLE, which may partially explain why mortality in ALP is $\sim 50 \%$ even when treated with large doses of steroids (14). Emergency surgery has been shown to be an independent risk factor for increasing the complication rate and morbidity of SLE patients $(15,16)$. In the present case, although the SLEDAI score was 10, ALP occurred, which may be explained by the emergency surgery performed for IpsO. The trauma of abdominal operation enhances inflammatory function, which may have promoted the activity of autoimmunity in SLE patients and eventually caused the occurrence of ALP.

The recommended therapy for ALP is high-dose methylprednisolone with gamma globulin supplementation. Early treatment with corticosteroids may significantly attenuate vascular injury. In the present case, the patient's clinical symptoms were ameliorated and the radiographic results showed improvement following high-dose methylprednisolone + gamma globulin supplement therapy for 3 days followed by $2 \mathrm{mg} / \mathrm{kg}$ methylprednisolone as maintenance treatment.

In conclusion, we report the first case of ALP and IpsO co-existing in a patient with SLE. It remains a challenge for the surgeon to establish the correct diagnosis and prevent inappropriate surgical intervention when IpsO plays a role in the initial presentation of SLE. ALP typically occurs during the active stage of SLE, thus emergency surgery may increase the rate of this complication. Early immunosuppressive therapy could be lifesaving in these patients.

\section{Acknowledgements}

This study was supported by the Natural Science Foundation of Zhejiang Province (grant. no. LY14H160033).

\section{References}

1. Kansal A, Jain A, Thenozhi S and Agarwal V: Intestinal pseudo-obstruction associated with biliary tract dilatation in a patient with systemic lupus erythematosus. Lupus 22: 87-91, 2013.

2. Ceccato F, Salas A, Góngora V, Ruta S, Roverano S, Marcos JC, Garcìa M and Paira S: Chronic intestinal pseudo-obstruction in patients with systemic lupus erythematosus: Report of four cases. Clin Rheumatol 27: 399-402, 2008.

3. Khairullah S, Jasmin R, Yahya F, Cheah TE, Ng CT and Sockalingam S: Chronic intestinal pseudo-obstruction: A rare first manifestation of systemic lupus erythematosus. Lupus 22: 957-960, 2013.

4. Chen YQ, Xue Q and Wang NS: Visceral muscle dysmotility syndrome in systemic lupus erythematosus: Case report and review of the literature. Rheumatol Int 32: 1701-1703, 2012.

5. Chaiamnuay S, Heck LW, Bell WC and Bastian HM: Acute granulomatous lupus pneumonitis: The first case report. Lupus 16: 201-204, 2007.

6. Cervera R, Khamashta MA, Font J, Sebastiani GD, Gil A, Lavilla P, Doménech I, Aydintug AO, Jedryka-Góral A, de Ramón E, et al: Systemic lupus erythematosus: Clinical and immunologic patterns of disease expression in a cohort of 1,000 patients. The European Working Party on Systemic Lupus Erythematosus. Medicine (Baltimore) 72: 113-124, 1993.

7. Sarkar S and Saha K: Bilateral acute lupus pneumonitis in a case of rhupus syndrome. Lung India 29: 280-282, 2012.

8. Hochberg MC: Updating the American College of Rheumatology revised criteria for the classification of systemic lupus erythematosus. Arthritis Rheum 40: 1725, 1997.

9. Cacoub P, Benhamou Y, Barbet P, Piette JC, Le Cae A, Chaussade S, Cadranel JF, Callard P, Opolon P and Godeau P: Systemic lupus erythematosus and chronic intestinal pseudoobstruction. J Rheumatol 20: 377-381, 1993.

10. Mok MY, Wong RW and Lau CS: Intestinal pseudo-obstruction in systemic lupus erythematosus: An uncommon but important clinical manifestation. Lupus 9: 11-18, 2000. 
11. Park FD, Lee JK, Madduri GD and Ghosh P: Generalized megaviscera of lupus: Refractory intestinal pseudo-obstruction ureterohydronephrosis and megacholedochus. World J Gastroenterol 15: 3555-3559, 2009.

12. Hill PA, Dwyer KM and Power DA: Chronic intestinal pseudo-obstruction in systemic lupus erythematosus due to intestinal smooth muscle myopathy. Lupus 9: 458-463, 2000.

13. Matthay RA, Schwarz MI, Petty TL, Stanford RE, Gupta RC, Sahn SA and Steigerwald JC: Pulmonary manifestations of systemic lupus erythematosus: Review of twelve cases of acute lupus pneumonitis. Medicine (Baltimore) 54: 397-409, 1975.
14. Urowitz MB, Bookman AA, Koehler BE, Gordon DA, Smythe HA and Ogryzlo MA: The bimodal mortality pattern of systemic lupus erythematosus. Am J Med 60: 221-225, 1976.

15. Koh ET, Seow A, Leong KH and Chng HH: SLE mortality in an oriental population. Lupus 6: 27-31, 1997.

16. Papa MZ, Shiloni E, Vetto JT, Kastner DL and McDonald HD: Surgical morbidity in patients with systemic lupus erythematosus. Am J Surg 157: 295-298, 1989. 